Pacific Journal of Mathematics

CHARACTERIZATIONS OF AMENABLE AND STRONGLY 


\title{
CHARACTERIZATIONS OF AMENABLE AND STRONGLY AMENABLE $C^{*}$-ALGEBRAS
}

\section{JOHN BuNCE}

\begin{abstract}
In this paper it is proved that a $C^{*}$-algebra $A$ is strongly amenable iff $A$ satisfies a certain fixed point property when acting on a compact convex set, or iff a certain Hahn-Banach type extension theorem is true for all Banach $A$-modules. It is proved that a $C^{*}$-algebra $A$ is amenable iff $A$ satisfies a weaker Hahn-Banach type extension theorem.
\end{abstract}

A topological group $G$ is said to be amenable if there is a left invariant mean on the space of bounded continuous complex functions on $G$. A number of papers have been published which give equivalent definitions of amenability (for example, see the papers $[4,7,11]$ or the book [3]). It has recently been proven that a locally compact group $G$ is amenable iff for all two-sided $L^{1}(G)$-modules $X$ and bounded derivations $D$ of $L^{1}(G)$ into $X^{*}$, we have that $D$ is the inner derivation induced by an element of $X^{*}$ [5, Theorem 2.5]. This result motivates the definition of amenable and strongly amenable $C^{*}$-algebras [5, sections 5 and 7]. In $\S 2$ of this paper we give some conditions on a $C^{*}$-algebra that are equivalent to amenability or strong amenability and are analogous to some of the known equivalent definitions of amenable groups. In $\S 3$ we show that the generalized StoneWeierstrass theorem for separable $C^{*}$-algebras is true when the $C^{*}$-subalgebra in question is strongly amenable.

1. Preliminaries. Let $A$ be a $C^{*}$-algebra. Then a complex Banach space $X$ is called a Banach $A$-module if it is a two-sided $A$-module and there exists a positive real number $M$ such that for all $a \in A$ and $x \in X$ we have

$$
\|a x\| \leqq M\|a\|\|x\|
$$

and

$$
\|x a\| \leqq M\|x\|\|a\| \text {. }
$$

If $X$ is a Banach $A$-module, then the dual space $X^{*}$ becomes a Banach $A$-module if we define for $a \in A, f \in X^{*}$, and $x \in X$,

$$
\begin{aligned}
& (a f)(x)=f(x a) \\
& (f a)(x)=f(a x) .
\end{aligned}
$$

A derivation from $A$ into $X^{*}$ is a bounded linear map $D$ from $A$ into 
$X^{*}$ such that $D(a b)=a D(b)+D(a) b$ for all $a, b \in A$. If $f \in X^{*}$, the function $\delta(f)$ from $A$ into $X^{*}$ given by

$$
\delta(f)(a)=a f-f a
$$

is called the inner derivation induced by $f$.

Definition 1. [5, §5]. A $C^{*}$-algebra $A$ is said to be amenable if every derivation from $A$ into $X^{*}$ is inner for all Banach $A$-modules $X$.

Definition 2. [5, §7]. A $C^{*}$-algebra $A$ is said to be strongly amenable if, whenever $X$ is a Banach $A$-module and $D$ is a derivation of $A$ into $X^{*}$, there is a $f \in \operatorname{co}\left\{D(u) u^{*}: u \in U\left(A_{e}\right)\right\}$ with $D=-\delta(f)$, where $A_{e}$ is the $C^{*}$-algebra obtained by adjoining the identity $e$ to $A, X$ is made into a unital $A_{e}$-module by defining $x e=e x=x$ for all $x \in K, D$ is extended to $A_{e}$ by defining $D(e)=0, U\left(A_{e}\right)$ is the unitary group of $A_{e}$, and co $S$ denotes the $w^{*}$-closed convex hull of a set $S$ contained in $X^{*}$.

A $C^{*}$-algebra $A$ is strongly amenable iff $A_{e}$ is strongly amenable, and a $C^{*}$-algebra $A$ with identity is strongly amenable iff the definition is satisfied for all unital $A$-modules $X$ with $A_{e}$ replaced throughout by $A[5, \S 7]$. The class of strongly amenable $C^{*}$-algebras includes all $C^{*}$-algebras which are $G C R$, uniformly hyperfinite, or the $C^{*}$-group algebra of a locally compact amenable group [5, §7]. It is not known if there exist amenable $C^{*}$-algebras which are not strongly amenable.

For $A$ a $C^{*}$-algebra, let $A \hat{\otimes} A$ be the completion of the algebraic tensor product $A \otimes A$ in the greatest cross-norm. Then we can identify $(A \otimes A)^{*}$ with the space of bounded bilinear functionals on $A \times A[13, \mathrm{p} .438]$. We see that $A \hat{\otimes} A$ becomes a Banach $A$-module if we define for $a, b, c \in A$,

$$
\begin{aligned}
& a(b \otimes c)=a b \otimes c \\
& (b \otimes c) a=b \otimes c a .
\end{aligned}
$$

Hence $(A \hat{\otimes} A)^{*}$ becomes a Banach $A$-module under the dual action: If $f \in(A \hat{\otimes} A)$ and $a, b, c \in A$,

$$
\begin{aligned}
& (a f)(b \otimes c)=f(b \otimes c a) \\
& (f a)(b \otimes c)=f(a b \otimes c) .
\end{aligned}
$$

We can also make $A \hat{\otimes} A$ and $(A \hat{\otimes} A)^{*}$ into Banach $A$-modules by defining for $f \in(A \hat{\otimes} A)^{*}$ and $a, b, c \in A$ :

$$
\begin{aligned}
& a \circ(b \otimes c)=b \otimes a c \\
& (b \otimes c) \circ a=b a \otimes c
\end{aligned}
$$




$$
\begin{aligned}
& (a \circ f)(b \otimes c)=f(b a \otimes c) \\
& (f \circ a)(b \otimes c)=f(b \otimes a c)
\end{aligned}
$$

Note that the two operations on $A \hat{\otimes} A$ do not interact; that is, if $a, b, c, d \in A$,

$$
\begin{aligned}
& a \circ(b(c \otimes d))=b(a \circ(c \otimes d)) \\
& ((c \otimes d) b) \circ a=((c \otimes d) \circ a) b \\
& a \circ((c \otimes d) b)=(a \circ(c \otimes d)) b
\end{aligned}
$$

and so forth.

\section{Amenable and strongly amenable $C^{*}$-algebras.}

Proposition 1. Let $A$ be a $C^{*}$-algebra with unit e. Then the following seven statements are equivalent:

(a) $A$ is strongly amenable.

(b) For all unital Banach $A$-modules $X$ and $f \in X^{*}$, there exists $g \in \operatorname{co}\left\{u f u^{*}: u \in U(A)\right\}$ such that $a g=g a$ for all $a \in A$.

(c) For any $f \in(A \widehat{\otimes} A)^{*}$ there exists $g \in \operatorname{co}\left\{u f u^{*}: u \in U(A)\right\}$ such that $a g=g a$ for all $a \in A$.

(d) There is a linear map $T$ of $(A \hat{\otimes} A)^{*}$ into $C=\left\{g \in(A \hat{\otimes} A)^{*}\right.$ : $a g=g a$ all $a \in A\}$ such that $T(a \circ f)=a \circ T(f), T(f \circ a)=T(f) \circ a$, and $T(f) \in \operatorname{co}\left\{u f u^{*}: u \in U(A)\right\}$ for all $a \in A, f \in(A \hat{\otimes} A)^{*}$.

(e) Let $X$ be a Banach A-module, $S$ a $w^{*}$-closed convex subset of $X^{*}$ such that $u s u^{*} \in S$ for all $s \in S, u \in U(A)$. Then there exists an element $s \in S$ such that $u s u^{*}=s$ for all $u \in U(A)$.

(f) Let $Y$ be a Banach A-module and $X$ a subspace of $Y$ such that $u x u^{*} \in X$ for all $x \in X, u \in U(A)$. Let $f \in X^{*}$ be such that $f\left(u x u^{*}\right)=$ $f(x)$ for all $x \in X, u \in U(A)$. Then for any $g \in Y^{*}$ which extends $f$, there is an $h \in \operatorname{co}\left\{u g u^{*}: u \in U(A)\right\}$ such that $h$ extends $f$ and $h\left(u y u^{*}\right)=$ $h(y)$ for all $y \in Y$ and $u \in U(A)$.

(g) Let $Y$ be a Banach $A$-module and $X$ a two-sided $A$-submodule of $Y$. Let $f \in X^{*}$ be such that $f\left(u x u^{*}\right)=f(x)$ for all $x \in X, u \in U(A)$. Then for any $g \in Y^{*}$ which extends $f$, there is an $h \in \operatorname{co}\left\{u g u^{*}: u \in U(A)\right\}$ such that $h$ extends $f$ and $h\left(u y u^{*}\right)=h(y)$ for all $y \in Y$ and $u \in U(A)$.

Before proving the proposition, we make some remarks. The implications (a) implies (b) and (b) implies (d) were proven in [1]. The map $T$ in (d) takes the place of the invariant mean that is present in amenable groups. The condition in (e) is a fixed point property; it is known that a locally compact group is amenable iff it has a certain fixed point property [7]. The condition (f) and (g) might be called the strong invariant extension property for subspaces, 
and the strong invariant extension property for submodules respectively. A locally compact group is amenable iff it has a certain HahnBanach type extension property similar to (f) and (g) [11].

Proof of Proposition 1. (a) implies (b): Let $f \in X^{*}$ and $\delta(f)$ be the inner derivation induced by $f$. Then there is a $a \in \operatorname{co}\left\{\delta(f)(u) u^{*}: u \in\right.$ $U(A)\}$ such that $\delta(f)=-\delta(g)$. But $\delta(f)(u) u^{*}=u f u^{*}-f$, hence $f+$ $g \in \operatorname{co}\left\{u f u^{*}: u \in U(A)\right\}$. Also $\delta(f)(a)=-\delta(g)(a)$ for all $a \in A$, thus $(f+g) a=a(f+g)$ for all $a \in A$.

(b) implies (c): Clear.

(c) implies $(d)$ : The proof is an adaption to the present situation of a proof of J. Schwartz [10, Lemma 5]. Let $A$ be the set of all linear mappings $T$ of $(A \hat{\otimes} A)^{*}$ into $(A \hat{\otimes} A)^{*}$ such that $T(f) \in \operatorname{co}\left\{u f u^{*}\right.$ : $u \in U(A)\}$ and $T(\alpha \circ f)=a \circ T(f), T(f \circ \alpha)=T(f) \circ a$ for all $f \in(A \hat{\otimes} A)^{*}$, $a \in A$. The set $\Lambda$ is nonempty since the identity map is in $\Lambda$. We order $\Lambda$ by defining $T_{1} \geqq T_{2}$ if for all $f \in(A \widehat{\otimes} A)^{*}$,

$$
\text { co }\left\{u T_{1}(f) u^{*}: u \in U(A)\right\} \subseteq \operatorname{co}\left\{u T_{2}(f) u^{*}: u \in U(A)\right\} \text {. }
$$

Then $\geqq$ defines a quasi-order on 4 . Suppose $\left\{T_{\alpha}: \alpha \in \Delta\right\}$ is a totally ordered subset of $\Lambda$. We have $\left\|T_{\alpha}(f)\right\| \leqq\|f\|$, thus for all $d \in A \widehat{\otimes} A$, $\left|T_{\alpha}(f)(d)\right| \leqq\|f\|\|d\|$ and $\left\{T_{\alpha}(f)(d): \alpha \in \Delta\right\}$ is a bounded function on $\Delta$. Let LIM be a Banach limit on the directed set $\Delta$ (see $[10$, p. 21] for information on Banach limits). Then set $T(f)(d)=\operatorname{LIM} T_{\alpha}(f)(d)$ for all $f \in(A \hat{\otimes} A)^{*}$ and $d \in A \hat{\otimes} A$. Then $T$ is a bounded linear map from $(A \hat{\otimes} A)^{*}$ into $(A \widehat{\otimes} A)^{*}$. An easy calculation shows that $T(a \circ f)=$ $a \circ T(f)$ and $T(f \circ a)=T(f) \circ a$. We show that $T(f) \in \operatorname{co}\left\{u f u^{*}: u \in U(A)\right\}$ and $T \geqq T_{\alpha}$ for all $\alpha \in \Delta$. If $\beta \geqq \alpha$ and $f \in(A \widehat{\otimes} A)^{*}$ then

$$
T_{\beta}(f) \in \operatorname{co}\left\{u T_{\alpha}(f) u^{*}: u U(A)\right\}=K .
$$

Suppose, for contradiction, that $T(f) \notin K$. Then by the strong separation theorem, there exists $d \in A \hat{\otimes} A, \lambda$ real and $\varepsilon>0$ such that for all $g \in K$,

$$
\operatorname{Re} T(f)(d) \leqq \lambda<\lambda+\varepsilon \leqq \operatorname{Re} g(d) .
$$

Hence $\operatorname{Re} T(f)(d) \leqq \lambda<\lambda+\varepsilon \leqq \operatorname{Re} T_{\beta}(f)(d)$ for all $\beta \geqq \alpha$. But applying the Banach limit to this equation we obtain $\operatorname{Re} T(f)(d)<\operatorname{Re} T(f)(d)$. Hence $T(f) \in K$. Thus

$$
\operatorname{co}\left\{u T(f) u^{*}: u \in U(A)\right\} \leqq \operatorname{co}\left\{u T_{\alpha}(f) u^{*}: u \in U(A)\right\}
$$

for all $\alpha \in \Delta$. Hence $T \in \Lambda$ and $T \geqq T_{\alpha}$. Hence $\Lambda$ is inductive, so by Zorn's lemma $\Lambda$ has a maximal element $T$. We show that $T(f) \in C$ for all $f \in(A \hat{\otimes} A)^{*}$. If $g \in(A \hat{\otimes} A)^{*}$ is such that $T(g) \notin C$, then co $\left\{u T(g) u^{*}: u \in U(A)\right\}$ contains more than one element. Since we 
assumed (c), $C \cap \operatorname{co}\left\{u T(g) u^{*}: u \in U(A)\right\}$ is nonempty. Let $\sum \lambda u T(g) u^{*}$ be a net indexed by a directed set $\Delta$ which converge $w^{*}$ to an element $h$ of $C$ (we suppress all indices in the sum). Define for $f \in(A \widehat{\otimes} A)^{*}$ and $d \in(A \hat{\otimes} A)$,

$$
T^{\prime}(f)(d)=\operatorname{LIM} \sum \lambda u T(f) u^{*}(d) \text {. }
$$

Then $T^{\prime}$ is a bounded linear map from $(A \hat{\otimes} A)^{*}$ to $(A \hat{\otimes} A)^{*}$ and another application of the strong separation theorem shows that $T^{\prime}(f) \in \operatorname{co}\left\{u T(f) u^{*}: u \in U(A)\right\}$. If we show that $T^{\prime}(\alpha \circ f)=\alpha \circ T^{\prime \prime}(f)$ and $T^{\prime \prime}(f \circ a)=T^{\prime}(f) \circ a$, we will know that $T^{\prime \prime} \in \Lambda$ and $T^{\prime} \geqq T$. But

$$
\begin{aligned}
T^{\prime}(a \circ f)(b \otimes c) & =\operatorname{LIM} \sum \lambda u T(a \circ f) u^{*}(b \otimes c) \\
& =\operatorname{LIM} \sum \lambda(a \circ T(f))\left(u^{*} b \otimes c u\right) \\
& =\operatorname{LIM} \sum \lambda T(f)\left(u^{*} b a \otimes c u\right) \\
& =T^{\prime}(f)(b a \otimes c) \\
& =\left(a \circ T^{\prime}(f)\right)(b \otimes c) .
\end{aligned}
$$

Hence $T^{\prime \prime}(a \circ f)=a \circ T^{\prime}(f)$ and likewise $T^{\prime}(f \circ a)=T^{\prime}(f) \circ a$. But the net $\sum \lambda u T(g) u^{*}(d)$ has the actual limit $h(d)$. Thus $T^{\prime}(g)=h$ and co $\left\{u T^{\prime \prime}(g)^{\prime}(g) u^{*}: u \in U(A)\right\}=\{h\}$, and $\{h\}$ is properly contained in co $\left\{u T(g) u^{*}: u \in U(A)\right\}$, hence it is not true that $T \geqq T^{\prime}$. But this contradicts the maximality of $T$, and we have that $T(f) \in C$ for all $f$. The completes the proof.

(d) implies $(e)$ : Let $x \in X$ and fix $s \in S$. Define a bounded bilinear function $F(x, s)$ on $A \times A$ by $F(x, s)(a, b)=s(a x b)$ for all $a, b \in A$. We consider $F(x, s)$ as an element of $(A \widehat{\otimes} A)^{*}$. Let $G(s)(x)=T(F(x, s))(e \otimes e)$. Then clearly $G(s) \in X^{*}$. Now if $u \in U(A)$, then

$$
\begin{aligned}
F\left(u^{*} x u, s\right)(a \otimes b) & =s\left(a u^{*} x u b\right) \\
& =F(x, s)\left(u \circ(a \otimes b) \circ u^{*}\right) \\
& =\left(u^{*} \circ F(x, s) \circ u\right)(a \otimes b) .
\end{aligned}
$$

Thus $F\left(u^{*} x u, s\right)=u^{*} \circ F(x, s) \circ u$. Hence for all $x \in X$ and $u \in U(A)$, by using the properties of the map $T$,

$$
\begin{aligned}
\left(u G(s) u^{*}\right)(x) & =G(s)\left(u^{*} x u\right) \\
& =T\left(F\left(u^{*} x u, s\right)\right)(e \otimes e) \\
& =T\left(u^{*} \circ F(x, s) \circ u\right)(e \otimes e) \\
& =\left(u T(F(x, s)) u^{*}\right)(e \otimes e) \\
& =T(F(x, s))(e \otimes e) \\
& =G(s)(x) .
\end{aligned}
$$

Thus $u G(s) u^{*}=G(s)$ for all $u \in U(A)$. We will be done when we show that $G(s) \in S$. If $G(s) \notin S$, then there exists $x \in X$, a real number 
$\lambda$ and $\varepsilon>0$ such that for all $t \in S$ we have

$$
\operatorname{Re} G(s)(x) \leqq \lambda<\lambda+\varepsilon \leqq \operatorname{Re} t(x) .
$$

Now $T(F(x, s)) \in \operatorname{co}\left\{u F(x, s) u^{*}: u \in U(A)\right\}$, and $\left(u F(x, s) u^{*}\right)(e \otimes e)=$ $\left(u s u^{*}\right)(x)$. Since $u s u^{*} \in S$ for all $u \in U(A)$, this implies that $\operatorname{Re} G(s)(x) \geqq$ $\lambda+\varepsilon$. This contradiction proves that $G(s) \in S$.

(e) implies (f): Let $X, Y$ and $f \in X^{*}$ be as in (f). Let $g \in Y^{*}$ be any extension of $f$ and let $S=\operatorname{co}\left\{u g u^{*}: u \in U(A)\right\}$. Then $S$ is $w^{*}$-closed convex subset of $Y^{*}$ and if $s \in S$ then $u s u^{*} \in S$ for all $u \in U(A)$. Hence by (e) there is an element $h \in S$ such that $u h u^{*}=h$ for all $u \in U(A)$. Since $u X u^{*} \subseteq X$ for all $u \in U(A), f\left(u x u^{*}\right)=f(x)$ for all $x \in X$ and $g$ extends $f$, it is easily seen that $h$ extends $f$.

(f) implies $(g)$ : Clear.

(g) implies $(c)$ : Given $g \in(A \widehat{\otimes} A)^{*}$, let $Y=A \widehat{\otimes} A, X=\{0\}, f=0$ and apply $(g)$. Thus there is an $h \in \mathrm{co}\left\{u g u^{*} ; u \in U(A)\right\}$ such that $h\left(u y u^{*}\right)=h(y)$ for all $y \in A \hat{\otimes} A$ and $u \in A$; that is, $a h=h a$ for all $a$ in $A$.

(d) implies (a): Let $D: A \rightarrow X^{*}$ be a derivation. Let $x \in X$ and define a bounded bilinear functional $f(x)$ on $A \times A$ by $f(x)(b, c)=$ $D(b)(x c)$. Then define an element $G \in X^{*}$ by $G(x)=T(f(x))(e \otimes e)$. We will show that $D=\delta(G)$ and $-G \in \operatorname{co}\left\{D(u) u^{*}: u \in U(A)\right\}$. For $x \in X$ and $a \in A$, define a bounded bilinear functional $g(x, a)$ on $A \times A$ by $g(x, a)(b, c)=D(a)(x c b)$. Then a computation shows that $a \circ f(x)=$ $f(a x)+g(x, a)$ and $f(x a)=f(x) \circ a$. If $a \in A, x \in X$, then

$$
\begin{aligned}
(\delta(G)(a))(x) & =(a G-G a)(x) \\
& =G(x a-a x) \\
& =T(f(x a-a x))(e \otimes e) \\
& =T(f(x) \circ a-a \circ f(x)+g(x, a))(e \otimes e) \\
& =T(f(x))(e \otimes a)-T(f(x))(a \otimes e)+T(g(x, a))(e \otimes e) \\
& =T(g(x, a))(e \otimes e) .
\end{aligned}
$$

The last equality is true because $T$ maps into $C$. Now for $u \in U(A)$, $\left(u g(x, a) u^{*}\right)(e \otimes e)=g(x, a)\left(u^{*} \otimes u\right)=D(\alpha)(x)$, and $T(g(x, a))$ is in co $\left\{u g(x, a) u^{*}: u \in U(A)\right\}$, hence $(\delta(G)(a))(x)=D(a)(x)$ for all $x \in X$ and $a \in A$, thus $D=\delta(G)$. An application of the strong separation theorem shows that $-G \in \operatorname{co}\left\{D(u) u^{*}: u \in U(A)\right\}$. Thus (d) implies (a) and the proof of Proposition 1 is complete.

REMARKs. (1) The equivalence of (a) and (c) shows that, to 
check strong amenability of $A$, it is only necessary to consider the $A$-module $A \hat{\otimes} A$; this gives another proof of the Proposition 7.15 of [5].

(2) In the notation of [6], a $C^{*}$-algebra $A$ is amenable iff the first cohomology group $H_{c}^{1}\left(A, X^{*}\right)$ is zero for all Banach $A$-modules $X$. The reduction of dimension argument of $[5, \S 1$. a] then shows that all the cohomology groups $H_{c}^{n}\left(A, X^{*}\right)$ are zero. If $A$ is strongly amenable, then the proof of (d) implies (a) above can be changed to show directly that $H_{c}^{n}\left(A, X^{*}\right)$ is zero for all $n$ and all Banach $A$ modules $X$; this proof is similar to the proof of Theorem 3.3 in [6], with the map $T$ taking the place of the invariant mean which is present in that theorem.

(3) In [1] the author used the existence of the function $T$ to generalize the well-known Dixmier-Mackey theorem on amenable groups by proving that every continuous representation of a strongly amenable $C^{*}$-algebra on a Hibert space is similar to a ${ }^{*}$-representation. However, this fact can be proved in a more elementary fashion as follows: Let $A$ be a strongly amenable $C^{*}$-algebra and let $\pi$ be a continuous representation of $A$ as bounded operators on a Hilbert space $H$. It suffices to assume $A$ has an identity $e$ and $\pi(e)=I$. Make $B(H)$ into a Banach $A$-module by the operations $a T=\pi(a) T$, $T a=T \pi\left(a^{*}\right)^{*}$ for $a \in A, T \in B(H)$. Then $B(H)$ is the dual Banach $A$-module of the trace class operators. Define a bounded linear map $D$ of $A$ into $B(H)$ by $D(a)=\pi(a)-\pi\left(a^{*}\right)^{*}$. Then

$$
\begin{aligned}
a D(b)+D(a) b & =\pi(a)\left(\pi(b)-\pi\left(b^{*}\right)^{*}\right)+\left(\pi(a)-\pi\left(a^{*}\right)^{*}\right) \pi\left(b^{*}\right)^{*} \\
& =\pi(a b)-\pi\left((a b)^{*}\right)^{*} \\
& =D(a b) .
\end{aligned}
$$

Hence $D$ is a derivation. Thus, since $A$ is strongly amenable, there is an operator $T$ in $\operatorname{co}\left\{D(u) u^{*}: u \in U(A)\right\}$ such that $D=-\delta(T)$. Then for $a \in A, \pi(a)-\pi\left(a^{*}\right)^{*}=-a T+T a=T \pi\left(a^{*}\right)^{*}-\pi(a) T$, thus $\pi(a)(I+$ $T)=(I+T) \pi\left(a^{*}\right)^{*}$. We let $R=T+I$. Now $D(u) u^{*}=\pi(u) \pi(u)^{*}-I$, thus $R \in \operatorname{co}\left\{\pi(u) \pi(u)^{*}: u \in U(A)\right\}$. For $x \in H,\left(\pi(u) \pi(u)^{*} x, x\right)=\left\|\pi(u)^{*} x\right\|^{2}$, and $\|x\|^{2}=\left\|\pi\left(u^{*}\right)^{*} \pi(u)^{*} x\right\|^{2} \leqq\|\pi\|^{2}\left\|\pi(u)^{*} x\right\|^{2}$. Hence $\left(1 /\|\pi\|^{2}\right)\|x\|^{2} \leqq$ $\left\|\pi(u)^{*} x\right\|^{2}$. Thus $R$ is positive and invertible. Let $S$ be the positive square root of $R$. Then $\pi(a) S^{2}=S^{2} \pi\left(a^{*}\right)^{*}$, and $S^{-1} \pi(a) S=S \pi\left(a^{*}\right)^{*} S^{-1}$. If we define $\pi_{1}(a)=S^{-1} \pi(a) S$, then $\pi_{1}$ is clearly a representation of $A$, and $\pi_{1}\left(a^{*}\right)^{*}=\left(S^{-1} \pi\left(a^{*}\right) S\right)^{*}=S \pi\left(a^{*}\right)^{*} S^{-1}=S^{-1} \pi(a) S=\pi_{1}(a)$. Hence $\pi_{1}$ is a ${ }^{*}$-representation.

We now give some equivalent conditions for a $C^{*}$-algebra to be amenable.

Proposition 2. Let $A$ be a $C^{*}$-algebra with unit e. Then the 
following three statements are equivalent:

(a) $A$ is amenable.

(b) There is a bounded linear map $T$ of $(A \hat{\otimes} A)^{*}$ into $C=$ $\left\{f \in(A \widehat{\otimes} A)^{*}: a f=f a\right.$ all $\left.a \in A\right\}$ such that $T$ restricted to $C$ is the identity on $C$ and $T(\alpha \circ f)=a \circ T(f), T(f \circ a)=T(f) \circ a$ for all $a \in A$, $f \in(A \widehat{\otimes} A)^{*}$.

(c) Let $Y$ be a Banach $A$-module and $X$ a two sided $A$-submodule of $Y$. Let $f \in X^{*}$ be such that $f\left(u x u^{*}\right)=f(x)$ for all $x \in X, u \in U(A)$. Then there is a $h \in Y^{*}$ such that $h$ extends $f$ and $h\left(u y u^{*}\right)=h(y)$ for all $y \in Y, u \in U(A)$.

Proof. (a) implies $(b)$ : Let $Y=(A \hat{\otimes} A) * \hat{\otimes}(A \hat{\otimes} A)$ and let $Z$, $W$ and $X$ be as in the proof of (g) implies (d) of Proposition 1. Let $F \in Y^{*}$ be defined by $F(f \otimes t)=f(t)$ and let $D_{1}$ be the inner derivation induced by $F$. Then for $a \in A, f \in(A \hat{\otimes} A)^{*}$, and $t \in(A \hat{\otimes} A)$, $D_{1}(a)(f \otimes t)=(a f-f a)(t)$. Hence $D_{1}(a)$ is zero on $W$. A calculation using the fact that the two $A$-module operations on $(A \hat{\otimes} A)$ do not interact (see the comment at the end of $\S 1$ ) shows that $D_{1}(a)$ is zero on $Z$. Hence there is a map $D$ from $A$ into $(Y / X)^{*}$ given by $D($ a) $(\bar{y})=$ $D_{1}(a)(y)$, where $\bar{y}$ is the coset in $Y / X$ of an element $y \in Y$. It is easily seen that $D$ is a derivation, hence since $A$ is amenable there is an element $G_{1} \in(Y / X)^{*}$ such that $D=\delta\left(G_{1}\right)$. Let $G \in Y^{*}$ be defined by $G(y)=G_{1}(\bar{y})$ for all $y \in Y$. Define a bounded linear map $T_{1}$ from $(A \hat{\otimes} A)^{*}$ into $(A \hat{\otimes} A)^{*}$ by $T_{1}(f)(t)=G(f \otimes t)$ for all $f \in(A \hat{\otimes} A)^{*}$, $t \in(A \widehat{\otimes} A)$. Now $D(\alpha)=a G_{1}-G_{1} a$ for all $a \in A$, thus

$$
\begin{aligned}
D(a) \overline{(f \otimes t)} & =D_{1}(a)(f \otimes t) \\
& =(a f-f a)(t) \\
& =\left(a G_{1}-G_{1} a\right) \overline{(f \otimes t)} \\
& =G(f \otimes(t a-a t) \\
& =T_{1}(f)(t a-a t) \\
& =\left(a T_{1}(f)-T_{1}(f) a\right)(t) .
\end{aligned}
$$

Hence $(a f-f a)(t)=\left(a T_{1}(f)-T_{1}(f) a\right)(t)$ for all $\hat{t} \in(A \otimes A)$, and we thus have $f-T_{1}(f) \in C$. Let $T$ be the bounded linear map from $(A \hat{\otimes} A)^{*}$ into $C$ given by $T(f)=f-T_{1}(f)$. If $f \in C$, then $T_{1}(f)(t)=G_{1} \overline{(f \otimes t)}=0$, thus $T(f)=f$ if $f \in C$. Similarly, since $G$ is zero on $Z$, we have that $T(a \circ f)=a \circ T(f)$ and $T(f \circ a)=T(f) \circ a$. This completes the proof of (a) implies (b).

(b) implies (c): Let $Y$ be a Banach $A$-module, $X$ a submodule of $Y$, and let $f \in X^{*}$ be such that $f\left(u x u^{*}\right)=f(x)$ for all $x \in X, u \in U(A)$. Let $f_{1} \in Y^{*}$ be any extension of $f$ and for each $y \in Y$ define an element $F(y)$ of $(A \hat{\otimes} A)^{*}$ by $F(y)(a \otimes b)=f_{1}(a y b)$. Then let $h \in Y^{*}$ be defined 
by $h(y)=T(F(y))(e \otimes e)$. A calculation shows that for all $u \in U(A)$, $F\left(u^{*} y u\right)=u^{*} \circ F(y) \circ u$, so that $h\left(u^{*} y u\right)=h(y)$. Also, if $x \in X$, then it is easily seen that $F(x) \in C$, hence $h(x)=T(F(x))(e \otimes e)=F(x)(e \otimes e)=$ $f_{1}(x)=f(x)$. Thus $h$ has the desired properties.

(c) implies (b): The proof is essentially the same as the proof of (g) implies (d) in Proposition 1; we omit the details.

(b) implies (a): Again, the proof is essentially the same as the proof of (d) implies (a) in Proposition 1.

While we can not settle the question of whether every amenable $C^{*}$-algebra is strongly amenable, we think that the relationship between conditions (c) of Proposition 2 and conditions (f) and (g) of Proposition 1 may be useful in settling the question.

3. A Stone-Weierstrass type theorem. For $A$ a $C^{*}$-algebra, let $E S(A)$ be the set of pure states of $A$. Let $B$ be a $C^{*}$-subalgebra of $A$ which separates $E S(A) \cup\{0\}$. The generalized Stone-Weierstrass question for $C^{*}$-algebras [9, section 4.7] asks when is $A$ equal to $B$ ? Using a method introduced by Sakai [8], we can show that $A=B$ if $A$ is separable and $B$ is strongly amenable.

Proposition 3. Let $A$ be a separable $C^{*}$-algebra. If $B$ is a strongly amenable $C^{*}$-subalgebra of $A$ which separates $E S(A) \cup\{0\}$, then $A=B$.

Proof. By [8, Lemma 1] we can assume that $A$ has an identity which is also in $B$. Then as in [8, proof of Proposition 2] if $B \neq A$, there is a ${ }^{*}$-representation $\pi$ of $A$ on a separable Hilbert space such that $(\pi(B))^{\prime \prime} \neq(\pi(A))^{\prime \prime}$. Then by [12, Theorem 12.2] there is a Hilbert space $H$ and a von Neumann algebra $D \subseteq B(H)$ such that $D$ is *anti-isomorphic to $D^{\prime}$ and such that $(\pi(B))^{\prime \prime}$ is *-isomorphic to $D$ by a *-isomorphism $S$. Now *-anti-isomorphisms are clearly order isomorphisms and hence are ultraweakly continuous [2, A27]. Thus the image of $\pi(B)$ under the *-anti-isomorphism is weakly dense in $D^{\prime}$. It was proven in [5, Section 7] that the weak closure of any *representation of a strongly amenable $C^{*}$-algebra has Schwartz's Property $P$ [10, Definition 1]; essentially the same proof shows that the weak closure of any *-anti-representation of a strongly amenable $C^{*}$-algebra has Property P. Hence, the von Neumann algebra $D^{\prime}$ has Property $\mathrm{P}$. Thus by [10, Lemma 5] there is a linear norm-decreasing map $P$ from $B(H)$ onto $D$ which is the identity on $D$. Now consider $S$ as a *-representation of $(\pi(B))^{\prime \prime}$ on $H$, then by $[2,2.10 .2]$, there is 
a Hilbert space $K$ containing $H$ and a *-representation $T$ of $(\pi(A))^{\prime \prime}$ on $K$ such that $S(x)=T(x) \mid H$ for all $x \in(\pi(B))^{\prime \prime}$. Let $p$ be the projection of $K$ onto $H$, and define a linear norm-decreasing map $R$ from $B(K)$ onto $B(H)$ by $R y=p y \mid H$ for all $y \in B(K)$. Then $S^{-1} \circ P \circ R \circ T$ is a linear norm-decreasing map from $(\pi(A))^{\prime \prime}$ onto $(\pi(B))^{\prime \prime}$ which is the identity on $(\pi(B))^{\prime \prime}$. Then by [8, Theorem 1], we have that $\left(S^{-1} \circ P \circ R \circ T\right) x=x$ for all $x \in(\pi(A))^{\prime \prime}$. Hence $(\pi(A))^{\prime \prime}=(\pi(B))^{\prime \prime}$. This contradiction shows that $A=B$.

We remark that Sakai [9, 4.7.8] has proved Proposition 3 in the case when $B$ is the uniform closure of an increasing directed set of Type I $C^{*}$-subalgebras. The author does not know of an example of a strongly amenable $C^{*}$-algebra which is not the uniform closure of an increasing directed set of Type I $C^{*}$-subalgebras.

\section{REFERENCES}

1. John Bunce, Representations of strongly amenable $C^{*}$-algebras, Proc. Amer. Math. Soc., 32 (1972), 241-246.

2. J. Dixmier, Les $C^{*}$-Algèbres et leurs Representations, Gauthier-Villars, Paris, 1964.

3. F. P. Greenleaf, Invariant Means on Topological Groups, Van Nostrand, New York, 1969.

4. A. Hulanicki, Groups whose regular representation weakly contains all unitary representations, Studia Math., 24 (1964), 37-59.

5. B. E. Johnson, Cohomology in Banach algebras, Memoir Amer. Math. Soc., Number 127, 1927.

6. R. V. Kadison and J. R. Ringrose, Cohomology of operator algebras II. Extended coboundaries and the hyperfinite case, Ark. Mat. 9 (1971), 55-63.

7. N. W. Rickert, Amenable groups and groups with the fixed point property, Trans. Amer. Math. Soc., 127 (1967), 221-232.

8. S. Sakai, On the Stone-Weierstrass theorem of $C^{*}$-algebras, Tohoku Math. J., 22 (1970), 191-199.

9. — $C^{*-a l g e b r a s ~ a n d ~} W^{*}$-algebras, Springer-Verlag, New York, 1971.

10. J. T. Schwartz, Two finite non-hyperfinite, non-isomorphic factors, Comm. Pure. Appl. Math., 16 (1963), 19-26.

11. R. J. Silverman, Means on semigroups and the Hahn-Banach extension property, Trans. Amer. Math. Soc., 83 (1956), 222-237.

12. M. Takesaki, Tomita's Theory of Modular Hilbert Algebras and its Applications, Lecture notes in Mathematics, No. 128, Springer-Verlag, New York, 1970.

13. F. Treves, Topological Vector Spaces, Distributions and Kernels, Academic Press, New York, 1967.

Received September 27, 1971. This research was partially supported by an NSF grant.

UNIVERSITY OF KANSAS 


\section{PACIFIC JOURNAL OF MATHEMATICS}

\section{EDITORS}

\author{
H. SAMELSON \\ Stanford University \\ Stanford, California 94305 \\ C. R. HOBBY \\ University of Washington \\ Seattle, Washington 98105
}

\section{J. DugundjI}

Department of Mathematics University of Southern California Los Angeles, California 90007

\section{RICHARD ARENS}

University of California

Los Angeles, California 90024

\section{ASSOCIATE EDITORS}
E. F. BECKENBACH
B. H. NeumanN
F. WOLF
K. YosHIDA

\section{SUPPORTING INSTITUTIONS}

\author{
UNIVERSITY OF BRITISH COLUMBIA \\ CALIFORNIA INSTITUTE OF TECHNOLOGY \\ UNIVERSITY OF CALIFORNIA \\ MONTANA STATE UNIVERSITY \\ UNIVERSITY OF NEVADA \\ NEW MEXICO STATE UNIVERSITY \\ OREGON STATE UNIVERSITY \\ UNIVERSITY OF OREGON \\ OSAKA UNIVERSITY
}

\author{
UNIVERSITY OF SOUTHERN CALIFORNIA \\ STANFORD UNIVERSITY \\ UNIVERSITY OF TOKYO \\ UNIVERSITY OF UTAH \\ WASHINGTON STATE UNIVERSITY \\ UNIVERSITY OF WASHINGTON \\ ${ }^{*} \stackrel{*}{*}{ }^{*}$
AMERICAN MATHEMATICAL SOCIETY
NAVAL WEAPONS CENTER
}

The Supporting Institutions listed above contribute to the cost of publication of this Journal, but they are not owners or publishers and have no responsibility for its content or policies.

Mathematical papers intended for publication in the Pacific Journal of Mathematics should be in typed form or offset-reproduced, (not dittoed), double spaced with large margins. Underline Greek letters in red, German in green, and script in blue. The first paragraph or two must be capable of being used separately as a synopsis of the entire paper. The editorial "we" must not be used in the synopsis, and items of the bibliography should not be cited there unless absolutely necessary, in which case they must be identified by author and Journal, rather than by item number. Manuscripts, in duplicate if possible, may be sent to any one of the four editors. Please classify according to the scheme of Math. Rev. Index to Vol. 39. All other communications to the editors should be addressed to the managing editor, Richard Arens, University of California, Los Angeles, California, 90024.

50 reprints are provided free for each article; additional copies may be obtained at cost in multiples of 50 .

The Pacific Journal of Mathematics is published monthly. Effective with Volume 16 the price per volume (3 numbers) is $\$ 8.00$; single issues, $\$ 3.00$. Special price for current issues to individual faculty members of supporting institutions and to individual members of the American Mathematical Society: $\$ 4.00$ per volume; single issues $\$ 1.50$. Back numbers are available.

Subscriptions, orders for back numbers, and changes of address should be sent to Pacific Journal of Mathematics, 103 Highland Boulevard, Berke'ey, California, 94708.

PUBLISHED BY PACIFIC JOURNAL OF MATHEMATICS, A NON-PROFIT CORPORATION

Printed at Kokusai Bunken Insatsusha (International Academic Printing Co., Ltd.), 270, 3-chome Totsuka-cho, Shinjuku-ku, Tokyo 160, Japan. 


\section{Pacific Journal of Mathematics}

\section{Vol. 43, No. $3 \quad$ May, 1972}

Max K. Agoston, An obstruction to finding a fixed point free map on a manifold.... 543

Nadim A. Assad and William A. Kirk, Fixed point theorems for set-valued mappings

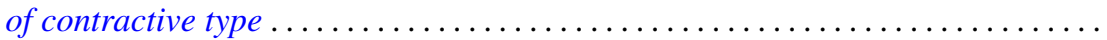

John Winston Bunce, Characterizations of amenable and strongly amenable

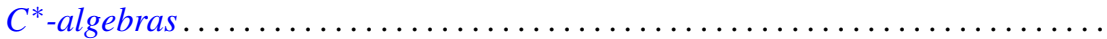

Erik Maurice Ellentuck and Alfred Berry Manaster, The decidability of a class of

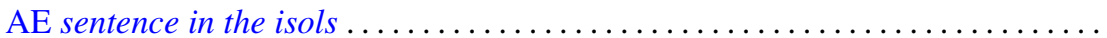

U. Haussmann, The inversion theorem and Plancherel's theorem in a Banach

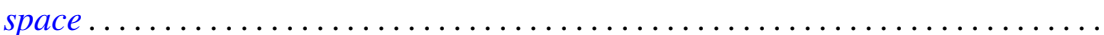

Peter Lawrence Falb and U. Haussmann, Bochner's theorem in infinite dimensions.

Peter Fletcher and William Lindgren, Quasi-uniformities with a transitive base ..... Dennis Garbanati and Robert Charles Thompson, Classes of unimodular abelian

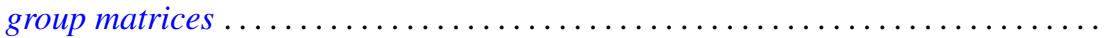

Kenneth Hardy and R. Grant Woods, On c-realcompact spaces and locally bounded normal functions

Manfred Knebusch, Alex I. Rosenberg and Roger P. Ware, Grothendieck and Witt rings of hermitian forms over Dedekind rings .......................

George M. Lewis, Cut loci of points at infinity.

Jerome Irving Malitz and William Nelson Reinhardt, A complete countable $L_{\omega_{1}}^{Q}$ theory with maximal models of many cardinalities . . . . . . . . . . . . . . . . .

Wilfred Dennis Pepe and William P. Ziemer, Slices, multiplicity, and Lebesgue

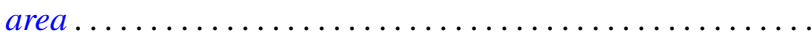

Keith Pierce, Amalgamating abelian ordered groups . .

Stephen James Pride, Residual properties of free groups . . . . . . . . . . . . . 725

Roy Martin Rakestraw, The convex cone of n-monotone functions .

T. Schwartzbauer, Entropy and approximation of measure preserving transformations .

Peter F. Stebe, Invariant functions of an iterative process for maximization of a polynomial...

Kondagunta Sundaresan and Wojbor Woyczynski, L-orthogonally scattered

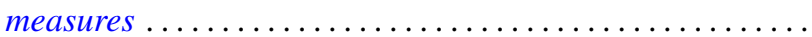

Kyle David Wallace, $C_{\lambda}$-groups and $\lambda$-basic subgroups $\ldots \ldots \ldots$

Barnet Mordecai Weinstock, Approximation by holomorphic functions on certain

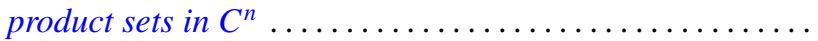

Donald Steven Passman, Corrections to: "Isomorphic groups and group rings”.

Don David Porter, Correction to: "Symplectic bordism, Stiefel-Whitney numbers, and a Novikov resolution"

John Ben Butler, Jr., Correction to: “Almost smooth perturbations of self-adjoint operators".

Constantine G. Lascarides, Correction to: "A study of certain sequence spaces of Maddox and a generalization of a theorem of Iyer" ...... ...

George A. Elliott, Correction to: "An extension of some results of takesaki in the reduction theory of von neumann algebras" ......................... 